\title{
Dispersion, an Important Radiation Mechanism for Ectomycorrhizal Fungi in Neotropical Lowland Forests?
}

\author{
Bernard Moyersoen \\ University of Aberdeen, School of Biological Sciences,
} UK

\section{Introduction}

Mycorrhizas are symbiotic associations between plant roots and fungi. They are divided into different categories depending on morphological characteristics and the identity of the fungi and plants (Smith \& Read, 2008). In tropical rainforests, most tree species are associated with arbuscular mycorrhizas (AM) (Alexander, 1989). Neotropical tree species belonging to confirmed ectomycorrhizal (EcM) host genera are diverse and generally scattered in a wide range of vegetation types (Table 1). Pakaraimaea dipterocarpacea, an endemic tree species from Guayana region (Maguire et al., 1977; Maguire \& Ashton, 1980; Moyersoen, 2006), is one of the few known locally dominant EcM tree species in the Neotropical lowland forests. This tree is phylogenetically related to the most important EcM tropical tree family in SE Asia: the Dipterocarpaceae. The disjunct distribution of $P$. dipterocarpacea suggests that the capacity to associate with EcM evolved in the ancestors of Dipterocarpaceae before the splitting of South America from Gondwana, c. 135 million year ago (Ma) (Moyersoen, 2006). There are alternative proposals to explain $P$. dipterocarpacea EcM status such as independent acquisition of EcM association in South America or a more recent transoceanic long-distance dispersal of EcM Dipterocarpaceae to the Neotropics (Alexander, 2006). Biotrophic fungi such as EcM can be an important source of information for improved understanding of disjunct distributions (Pirozynski, 1983). The phylogeny of P. dipterocarpacea EcM fungi might reflect the disjunct distribution of this endemic tree species. A pioneering survey on $P$. dipterocarpacea indicated that broad host range fungal lineages distributed across the tropics are associated with this host plant (Moyersoen, 2006), but no phylogeographic studies on $P$. dipterocarpacea EcM fungi are available to date.

Apart from human transport, there are three possible hypotheses to predict the phylogeography of EcM fungal community associated with $P$. dipterocarpacea, i.e. vicariance, dispersion and/or migration. Both migration and vicariance assume that there is a close and specialized relationship between the fungus and the plant partners in EcM symbiosis (Pirozynski, 1983; Halling et al., 2008). Any discovery of fungi with an old Gondwanan origin associated with $P$. dipterocarpacea would support the vicariance hypothesis. Radiations of EcM fungi across continents are explained by co-migrations of both the fungus and the host partners (Halling et al., 2008; Pirozynski, 1983) and the capacity of the fungi to switch 
hosts (Halling, 2001). The possible long term co-existence of Neotropical Dipterocarpaceae with other EcM host families in the same region (including the EcM Fabaceae Dicymbe) might have favored this radiation scenario (Moyersoen, 2006). Dispersion assumes that EcM fungi are able to cross environmental barriers, usually by spore dispersal. Long distance dispersion, presumably by trans-Tasman airflow, was proposed to explain the disjunct distribution of Pisolithus (Moyersoen et al., 2003) and Inocybe (Matheny et al., 2009) species between Australia and New Zealand. The biotrophic status of EcM fungi is an important constraint for the movement of these fungi and the relative importance of long distance dispersion versus migration and vicariance in global EcM fungi distribution is under debate (Halling et al., 2008).

\begin{tabular}{|c|c|c|c|c|c|}
\hline & \multirow{2}{*}{$\begin{array}{l}\text { Species } \\
\text { in } \mathrm{VG}^{*} \\
\text { (total } \\
\text { species) }\end{array}$} & \multicolumn{4}{|c|}{ For each entire genus } \\
\hline & & $\begin{array}{l}\text { Life } \\
\text { forms }\end{array}$ & Vegetation range & $\begin{array}{l}\text { Altitudinal } \\
\text { range }\end{array}$ & Geographical range \\
\hline \multicolumn{6}{|l|}{ Caesalpiniaceae } \\
\hline \multicolumn{6}{|l|}{ Amherstieae } \\
\hline Dicymbe & $11(19)$ & $\begin{array}{l}\text { Small to } \\
\text { large } \\
\text { trees }\end{array}$ & $\begin{array}{l}\text { Rainforests, shrublands, } \\
\text { shrublands on rocky } \\
\text { substrate, gallery } \\
\text { forests }\end{array}$ & 200-2700m & $\begin{array}{l}\text { Southwestern Colombia, } \\
\text { Southern Venezuela, } \\
\text { Guyana, Surinam, } \\
\text { Northwestern Brazil }\end{array}$ \\
\hline \multicolumn{6}{|l|}{ Fabaceae s.str. } \\
\hline Aldina & $18(22)$ & $\begin{array}{l}\text { Small to } \\
\text { large } \\
\text { trees }\end{array}$ & $\begin{array}{l}\text { Rainforests, shrublands, } \\
\text { gallery forests, white } \\
\text { sand shrub savannas, } \\
\text { forest-savanna ecotone, } \\
\text { savannas, riparian } \\
\text { forests, swamp forests, } \\
\text { shrublands on rocky } \\
\text { substrate }\end{array}$ & 100-1800m & $\begin{array}{l}\text { Southwestern Colombia, } \\
\text { Southern Venezuela, } \\
\text { Guyana, Northwestern } \\
\text { Brazil }\end{array}$ \\
\hline \multicolumn{6}{|c|}{ Dipterocarpaceae } \\
\hline Pakaraimaea & $1(1)$ & $\begin{array}{l}\text { Trees,or } \\
\text { shrubs }\end{array}$ & $\begin{array}{l}\text { Forests on sandy soil, } \\
\text { shrublands on rocky } \\
\text { slopes }\end{array}$ & $500-1100 \mathrm{~m}$ & $\begin{array}{l}\text { Southern Venezuela, } \\
\text { Guyana }\end{array}$ \\
\hline Pseudomonotes & $0(1)$ & Trees & $\begin{array}{l}\text { Rainforests on clayey to } \\
\text { sandy soil }\end{array}$ & $200-300 \mathrm{~m}$ & Southwestern Colombia \\
\hline \multicolumn{6}{|l|}{ Pisonieae } \\
\hline Guapira & $\begin{array}{l}13 \\
(\text { ca 50) }\end{array}$ & $\begin{array}{l}\text { Trees or } \\
\text { shrubs }\end{array}$ & $\begin{array}{l}\text { Evergreen, } \\
\text { semideciduous or } \\
\text { deciduous forests, } \\
\text { gallery forests, riparian } \\
\text { forests, savanna-dry } \\
\text { forest ecotone, white } \\
\text { sand shrublands }\end{array}$ & $50-1300 \mathrm{~m}$ & $\begin{array}{l}\text { Wide distribution from } \\
\text { Mexico, West Indies to } \\
\text { Brazil, Paraguay, and from } \\
\text { Peru, Ecuador to French } \\
\text { Guiana, Trinidad-Tobago }\end{array}$ \\
\hline Neea & $\begin{array}{l}30 \\
(\text { ca 85) }\end{array}$ & $\begin{array}{l}\text { Trees or } \\
\text { shrubs }\end{array}$ & $\begin{array}{l}\text { Rainforests, seasonally } \\
\text { dry evergreen forests, } \\
\text { semievergreen forests, } \\
\text { riparian forests, } \\
\text { savanna-dry forest } \\
\text { ecotone, white sand } \\
\text { savannas, white sand } \\
\text { shrublands, shrublands }\end{array}$ & $50-2000 \mathrm{~m}$ & Neotropics \\
\hline
\end{tabular}




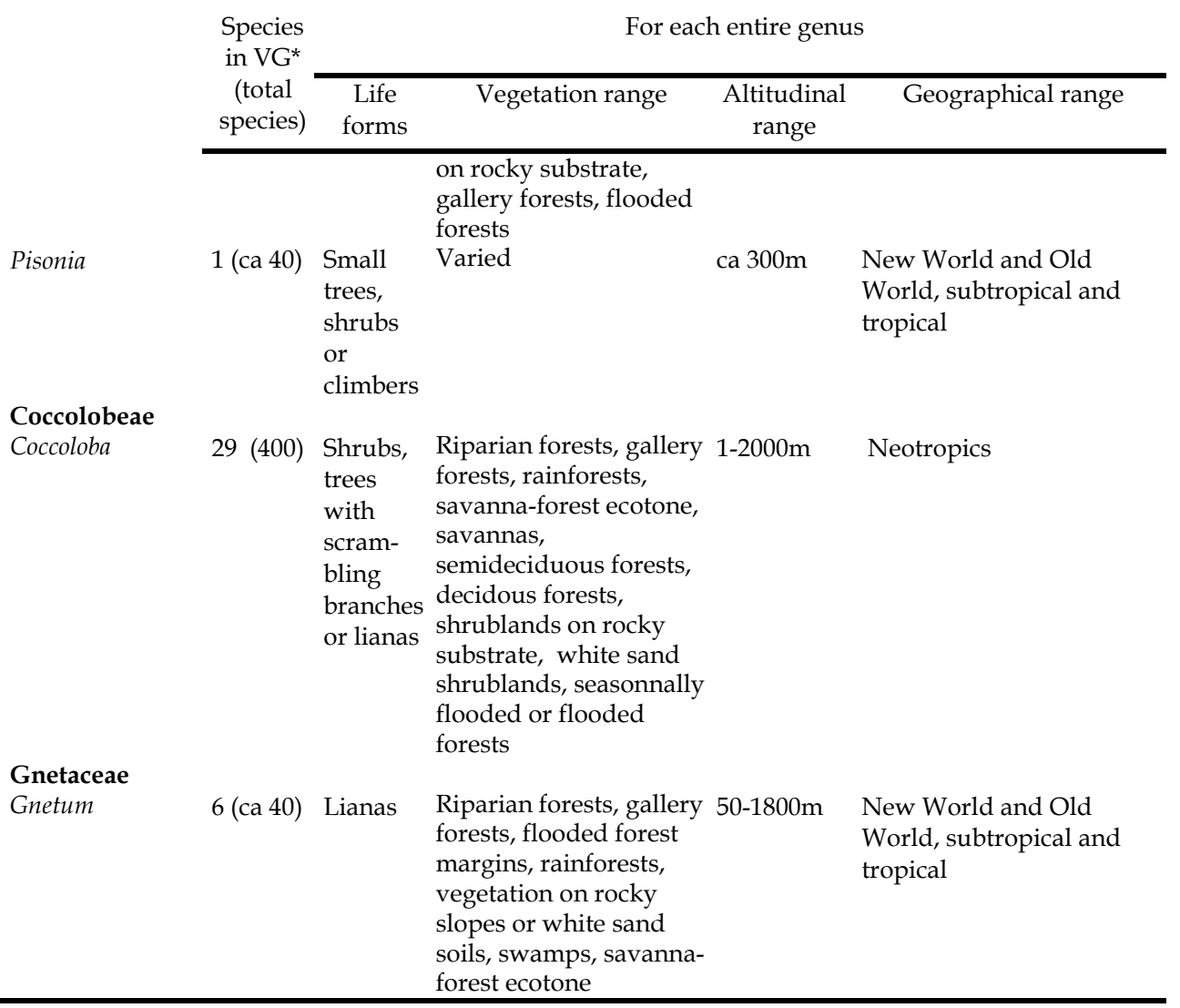

Table 1. Richness and distribution of confirmed EcM plant genera in the Neotropical lowland forests. *Venezuelan Guayana. Source : Steyermark et al. (1995)

The objective of this study was to evaluate the different hypotheses about the possible origin of EcM fungi associated with $P$. dipterocarpacea. For comparisons between $P$. dipterocarpacea $\mathrm{EcM}$ fungi and other tropical tree species in the same region or elsewhere, EcM fungi diversity and community structure was evaluated in a plot, where clumps of $P$. dipterocarpacea are present together with individuals of the EcM tree Aldina sp., Fabaceae, Papilionoïdeae. Inocybe was selected for further phylogenetic analysis, because of its global importance as an EcM fungal lineage, the general knowledge about its biogeography and its hypothesized paleotropical origin (Kuyper, 1986; Matheny et al., 2009; Ryberg, 2009).

A great diversity of EcM fungi, comparable to other tropical or temperate EcM rich communities, was found in this study. The EcM community structure was similar to that found in another forest dominated by EcM Fabaceae Dicymbe sp. in the same region. This study was the first evidence that Neotropical host tree species belonging to the Dipterocarpaceae and the EcM Fabaceae share EcM fungi both within the same forest and across forest stands. The floristic similarities and phylogenetic relationships of the EcM fungi between $P$. dipterocarpacea and Dicymbe forests suggested that fungal dispersion is an 
important radiation mechanism for EcM fungi in the region. Close phylogenetic relationships between Inocybe species associated with $P$. dipterocarpacea and African strains confirmed EcM phylogeographic links between the two continents. This study should be extended to examine a new $P$. dipterocarpacea dominated forest. The possible importance of dispersion in EcM fungal radiation and the link with paleotropical fungi should be tested in additional fungal lineages.

\section{Methods}

\subsection{Study site}

The sampling site was as described by Moyersoen (2006), located at $4^{\circ} 20^{\prime} \mathrm{N}, 61^{\circ} 48^{\prime} \mathrm{W}$, altitude 500 m, near Icabarú Village, in Gran Sabana, Estado Bolivar, Venezuela, (Fig. 1). The precise location of the $20 \times 20 \mathrm{~m}$ plot was selected based on a previous report of a stand of $P$. dipterocarpacea ssp. nitida described by Maguire \& Steyermark (1981) in the same area (Fig.2a). A second EcM tree, Aldina sp., also occurs in the plot (Moyersoen, 2006). Four separate sampling expeditions were conducted, in November 2003 (one day), March 2006 (one day), July 2007 (5 days) and July 2008 (9 days), respectively.

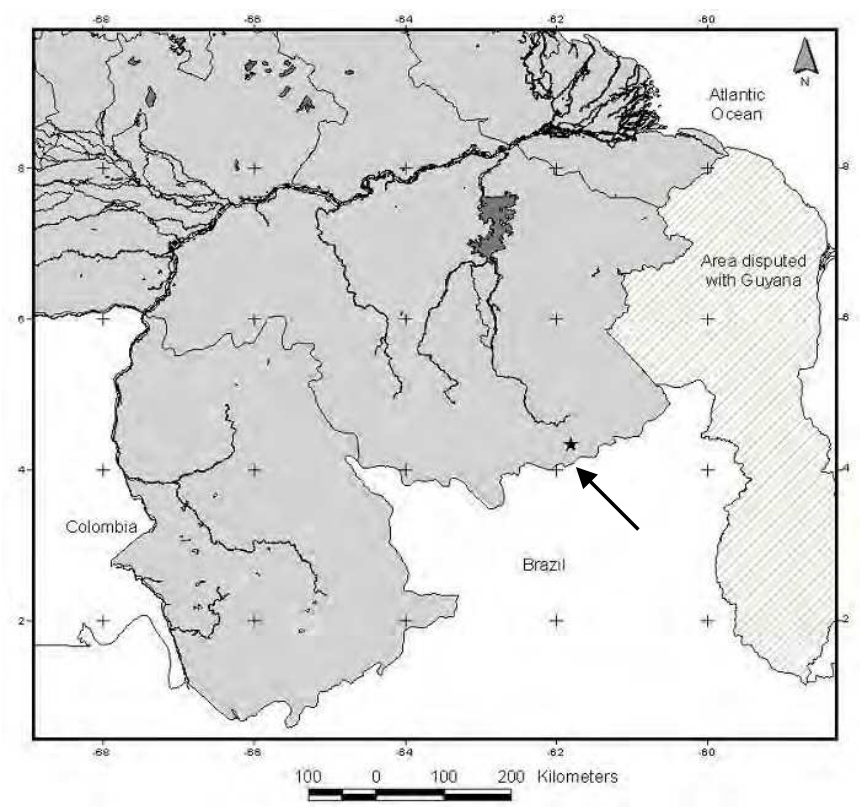

Fig. 1. Location of the field site

\subsection{Fruit body and fine root sampling}

All terricolous fruit bodies belonging to different morphotypes as well as resupinate fruit bodies growing under dead trunks and branches were collected inside the $20 \times 20 \mathrm{~m}$ plot in 2007 and 2008 (Fig. 2-b, c, d). A brief description from fresh material was made in the field for each collection and a piece of mycelium from the cap was taken and fixed in cetyltrimethylammonium bromide $(\mathrm{CTAB})$ for further molecular study. Time lapse between 
collection and fixation was less than one day. Microscopic observations of $3 \% \mathrm{KOH}$ rehydrated material was carried out using a light microscope (Leica DM 2500). Fruit body collections were assigned to families or genus using general keys and assigned to morphospecies. Fungi were considered to be putatively EcM on the basis of identification (e.g. Rinaldi et al., 2008; Singer, 1986; Smith \& Read, 2008).

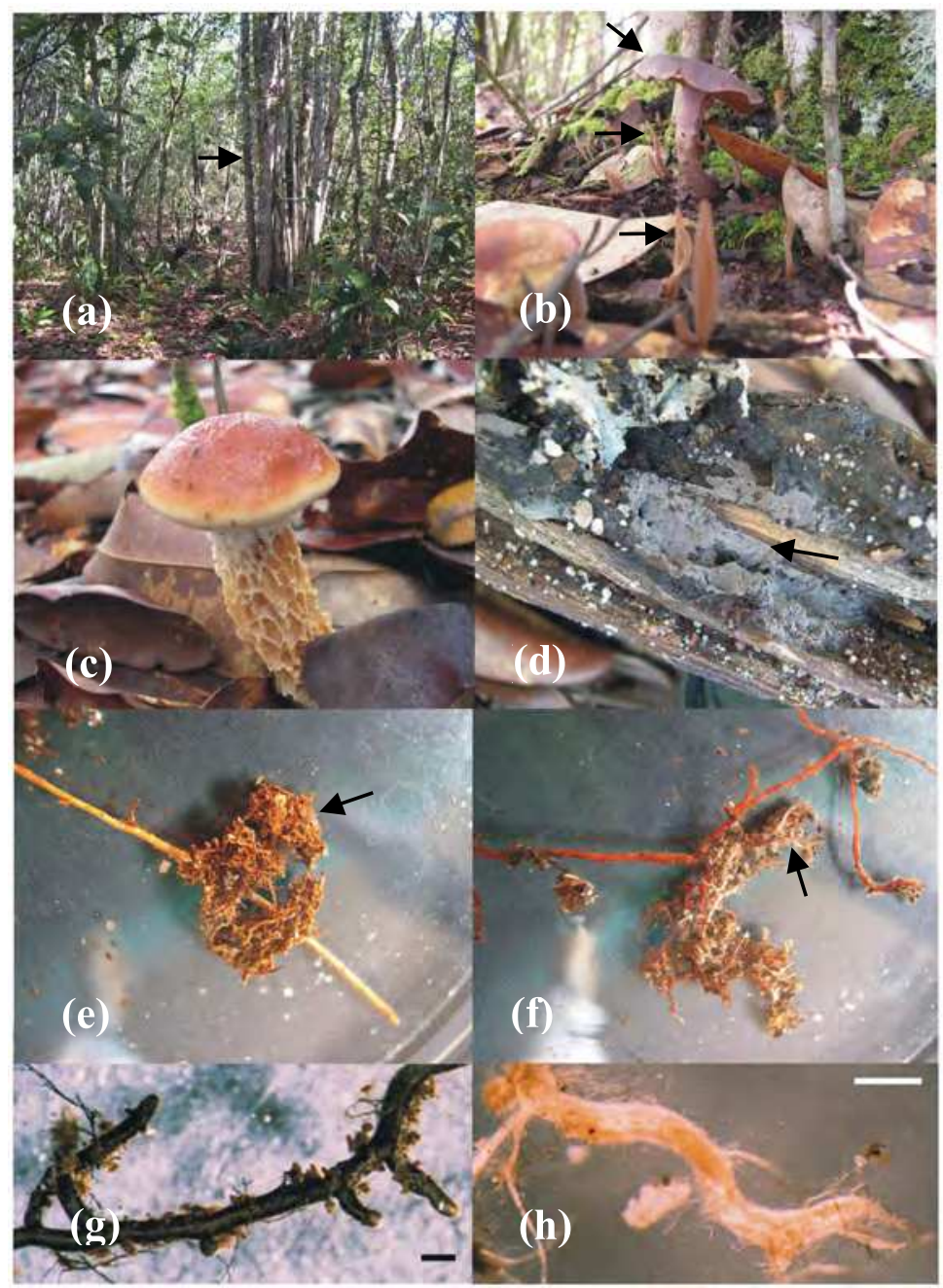

Fig. 2. Ectomycorrhizal (EcM) elements in Pakaraimaea dipterocarpacea forest plot. (a) Clump of $P$. dipterocarpacea; (b) Cortinarius sp. (BM08C30) surrounded by clavate Clavulina sp. (cf. BM07C9) fruit bodies; (c) Austroboletus rostrupii fruit body; (d) Resupinate Tomentella sp. (BM07C26, matching PD2_3) fruit body under dead trunk; (e) EcM system attached to Aldina sp. "B" secondary root (f) EcM system attached to P. dipterocarpacea secondary root ; (g) Tomentella sp. EcM (BM03M8, matching PD2_3) on Aldina sp.; Cortinarius sp. EcM (BM03M4, matching BM08C20) on P. dipterocarpacea. Bar, $0,4 \mathrm{~mm}$. 
Fine roots were sampled in each sampling expedition. They were traced from identified $P$. dipterocarpacae trees in the first three surveys and sampled in nine $10 \times 10 \times 5 \mathrm{~cm}$ deep soil cores scattered in the plot in the last harvest. The soil cores were located either in places where $P$ dipterocarpacea and Aldina $\mathrm{sp}$ trees occurred at $<2 \mathrm{~m}$ distance from each other (5 cores) or in random locations (4 cores).

Traced root samples were morphotyped using a field dissecting microscope (Novex AP-7) or a 10X magnifying glass before fixation in CTAB. Time lapse between tracing and root fixation was always up to one day. Morphology of traced $P$ dipterocarpacea and Aldina sp. fresh secondary roots was characterized for subsequent sorting in the soil cores. Color was rust brown to yellowish and silvery creamish in $P$. dipterocarpacea and Aldina sp., respectively (Fig. 2- e, f). Frequent longitudinal peels of bark were often observed on $P$. dipterocarpacea roots.

A $5 \times 5 \times 5 \mathrm{~cm}^{3}$ subsample including top organic soil was taken from each soil core and most fine roots attached to secondary roots as well as loose root tips were carefully washed in the field Lab. EcM and non-EcM root systems were separated using a 10X magnifying glass. EcM roots were subsequently separated on $P$. dipterocarpacea and Aldina root systems using color and morphological features. Loose EcM tips were grouped into a third "unclassified" category. All roots from the three categories were fixed in CTAB for further morpho-anatomical study in the laboratory in Belgium. Time lapse between root sampling and fixation was less than one day.

For each soil core, all fine roots belonging to P. dipterocarpacea, Aldina and "unclassified" categories were screened separately under a dissecting microscope to assign them to EcM morphological categories (Fig. 2-g, h). Each morphological category, including several tips, was cross checked using anatomical features on mantle peels following Agerer's (1991) method with some modifications. Different morphotypes from each P. dipterocarpacea, Aldina and "unclassified" root category were stored separately in CTAB and kept as a representative sample for further molecular analysis.

\subsection{DNA protocols}

Genomic DNA was extracted using QIAGEN Dneasy ${ }^{\mathrm{TM}}$ Plant Mini Kit (Qiagen S.A. Courtaboeuf, France) from fruit body morphospecies, traced ECM and morphotypes from the soil cores. To test the accuracy of morphotypes in the soil cores, DNA was extracted from several replicates (between two and 6) in EcM morphotypes including more than one sample. Fungal internal transcript spacer (ITS) and partial large subunit (LSU, 25-28S) nuclear rDNA were PCR-amplified with forward primer ITS1f in combination with LR6 (Gardes \& Bruns, 1993; Vilgalys \& Hester, 1990). If multiple or no PCR products were obtained, DNA was extracted from another EcM tip or the same extract was reamplified using the following primers in different combinations: ITS1f, ITS4b, ITS4, 5,8SR, LR21, LROR, LR6 (Gardes \& Bruns, 1993; Vilgalys \& Hester, 1990; White et al., 1990; R Vilgalys Lab http://www.biology.duke.edu/fungi/mycolab/primers.htm). The PCR protocols were as described in Moyersoen (2006) with some modifications in cycling parameters for different primers. To check host identity of traced roots and morphotypes from the soil cores, $r b c \mathrm{~L}$ DNA was amplified from 7 EcM samples of $P$. dipterocarpacea and Aldina sp. using the primers $r b c \mathrm{LN}$ and $r b c \mathrm{LR}$ (Käss \& Wink, 1997), following the same PCR protocols as in Moyersoen (2006). ITS-LSU and $r b c \mathrm{~L}$ amplification products were electrophoresed in 
$1 \%$ agarose gels stained with ethidium bromide and visualized under UV light. $1 \mathrm{~kb}+$ DNA ladder (Invitrogen) was used as a marker. Controls with no DNA were included in every set of PCR amplifications. PCR products were purified using QIAquick protocol (Qiagen) or 96well filtration system (Multiscreen-PCR plate, Millipore Corporation, MA, USA). Sequencing was performed by Genotranscriptomics Platform, GIGA, University of Liège , using the same primers as for PCR. Sequence editing was done using SEQUENCHER, version 4.0 (Gene Codes Corporation). ITS sequences of EcM specimens have been deposited at the National Center for Biotechnology Information (NCBI, GenBank: http:/ / www.ncbi.nlm.nih.gov) under accession numbers JQ063044-JQ063063. Sequences of new fruit body species will be published separately.

\subsection{Sequence analysis and phylogenetic analysis of Inocybe}

ITS sequences were assigned to molecular species on the basis of arbitrary $3 \%$ similarity cutoff value (Nilsson et al., 2008). Sequence similarities were determined using the BLASTN sequence similarity tool (Altschul et al., 1997) in GenBank together with BLAST comparisons with recently published ITS sequences from a Dicymbe forest kindly supplied by M Smith (Smith et al., 2011).

A preliminary phylogenetic analysis was performed to place Inocybe sequences in Inocybaceae clades (Matheny et al., 2009). 5' end nLSU sequences of two Inocybe species from $P$. dipterocarpacea forest were aligned using Clustal X (V. 2.0.9) to Matheny et al. (2009) global Inocybaceae sequences data set kindly supplied by the first author. Maximum likelihood (ML) analysis was implemented in the program RAxML V. 7.0.6, using GTRCAT approximation. Taxon sampling was made on the basis of this analysis. All strains in Inocybe subgenus Inocybe sequences and a strain from Nothocybe lineage were selected in Matheny et al. (2009) data base together with sequences from recently published tropical strains Y01 (UDB 004238), Y02 (UDB004239) (Tedersoo et al., 2010a) and I. tauensis (GU97711, GU977212, GU977213) (Kropp \& Albee-Scott, 2010). In total, the data set included 77 RNA polymerase II (RPB1), 68 RPB2 and 114 LSU sequences. The RPB1, RPB2 and nLSU sequences were aligned using Clustal X. For consistency, the same criteria as Matheny et al. $(2005,2009)$ were used for manual alignment using Bioedit V. Introns 1 of RPB1 and the intron of RPB2 were excluded from the analysis. Intron boundaries were inferred both by sequence comparison using published I. lilacina RPB1 intron 1 (AY351834), I. sindonia RPB1 intron 2 (AY351839) and by insertion between conserved amino acid and the canonical guanine-thymine and adenosine-guanine splice sites. Other positions, too ambiguous to align, were removed from the data set. RPB1 exon, RPB1 intron 2, RPB2 and nLSU were then concatenated in Bioedit. Data set partitioning was done following Matheny (2005). To test the phylogenetic relationships of Inocybe strains from $P$. dipterocarpacea forest with Inocybe data set, a ML Rapid Bootstrapping algorithm was implemented for 1000 replicates in RAxML, using GTRCAT approximation.

\section{Results}

\subsection{Above and below ground EcM richness}

A total of 64 EcM fruitbody samples were collected in the plot (Table 2). From these samples, 41 specimens (including replicates) were selected for molecular analysis and 38 (93\%) were successfully sequenced. These sequences belonged to 26 molecular species. Descriptions of new fruit body species will be published separately. 
From 15 adult trees and the soil cores, 150 EcM samples were recovered (Table 2). A total of 113 EcM samples were selected for molecular analysis and 97 EcM tips (86\%) were successfully amplified and sequenced. These sequences belonged to $27 \mathrm{EcM}$ fungal species. Only 12 of these species matched DNA from fruitbody surveys.

\begin{tabular}{llllllll} 
& Samples & PCR & $\begin{array}{l}\text { Total } \\
\text { sequences* }\end{array}$ & $\begin{array}{l}\text { Success } \\
\text { rate }(\%)\end{array}$ & $\begin{array}{l}\text { Inocybe } \\
\text { sequences }\end{array}$ & $\begin{array}{l}\text { Total } \\
\text { species }^{* *}\end{array}$ & $\begin{array}{l}\text { Inocybe } \\
\text { species }\end{array}$ \\
\hline Fruitbodies & 64 & 41 & 38 & 0.93 & 2 & 26 & 1 \\
EcM & 150 & 113 & 97 & 0.86 & 8 & 27 & 2 \\
Total & 214 & 154 & 135 & 0.88 & 10 & $38^{* * *}$ & 2 \\
\hline
\end{tabular}

*Including ITS1, 5.8S, ITS2, LSU DNA regions depending on species.

**Species were defined using $97 \%$ sequence similarity cutoff across the ITS1, 5.8S, ITS2 region as well as phylogenetic analysis of LSU on selected fungal groups.

***This figure does not include one unsequenced Russula and Scleroderma species.

Table 2. Observed fruitbody and EcM species in $400 \mathrm{~m}^{2}$ plot dominated by Pakaraimaea dipterocarpacea

EcM morphotypes accuracy was tested before further measurement of species density. A total of 96 EcM samples from soil cores were classified in 28 preliminary morphological categories. From these samples, 64 EcM were subject to molecular analysis, resulting in 56 (83\%) successful sequences and 22 species. Some preliminary categories were lumped together and 20 morphotypes were defined after cross molecular analysis. Only two morphotypes belonging to Cortinarius and Tomentella were accurate at genus level. The remaining morphotypes were accurate at the species level. Between one and 12 different morphotypes could be retrieved from a single $125 \mathrm{~cm}^{3}$ soil core (Table 3). Species density per soil core was similar or above values reported in an highly diverse temperate Picea abies and Tilia cordata dominated EcM community where similar sampling strategy was used (Table 3).

Overall, 40 fungal species were recovered in the plot. Comparison with surveys in similar plot size in other tropical regions or in temperate regions showed that observed species richness was great and similar to or greater than values both in temperate and tropical areas (Table 4).

\subsection{Host identity of ectomycorrhizal species}

Host identity of all 7 P. dipterocarpacea EcM samples including traced roots (three samples) and morphotypes from the soil cores was confirmed after molecular cross checking (305/308, 99\% similarity with P. dipterocarpacea, DQ406587). Among the 7 Aldina EcM samples (from soil cores), 5 tips matched A. latifolia, U74252 (99\%, 594/600) rbcL. The remaining two samples belonged to $P$. dipterocarpacea. Aldina leaf morphology corresponded to "species B" in Flora of Venezuelan Guayana (1995) (G Aymard, pers. com.). Sequenced EcM singletons observed on "unclassified" roots belonged to $P$. dipterocarpacea.

In total, 22 EcM fungal species were associated with P. dipterocarpacea; 11 (50\%) of these species belonging to 7 fungal groups were also putative or molecularly confirmed associates of Aldina, and three singletons were associated with Aldina only (Table 5). 


\subsection{EcM community composition and comparisons with other tropical forest communities}

The 40 fungal species were distributed in 7 fungal orders including Agaricales (11 species), Thelephorales (6 species), Cantharellales (6 species), Boletales (6 species), Russulales (5 species), Hymenochaetales (3 species) and Sebacinales (3 species) (Table 5). Agaricales were the richest fungal order including Cortinarius species (5 species), Amanitaceae (3 species), Inocybaceae ( 2 species) and one Tricholomataceae species (Table 5).

Eighty percent $(80 \%)$ of the best aligned sequences were from the tropical areas including both the Paleotropics and the Neotropics (Table 5). Only 13 (33\%) species matched ( $\geq 97 \%$ similarity) the published ITS sequences. These species belonged to Amanita, Inocybe, Boletaceae, Clavulina, Sebacinales and Tomentella. All but one of these species were reported from a Dicymbe dominated forest situated ca $240 \mathrm{kms}$ apart in the Guayana region (Guyana, Upper Potaro River Basin). Confirmed tree hosts included Dicymbe and Aldina species (Smith et al. 2011).

This study Morris et al. Tedersoo et al.Smith et al. Tedersoo et al. Morris et al.

\begin{tabular}{|c|c|c|c|c|c|c|}
\hline & & (2009) & (2010a) & (2011) & (2003) & (2008) \\
\hline Climate & $\begin{array}{l}\text { Humid } \\
\text { tropical }\end{array}$ & $\begin{array}{l}\text { Humid } \\
\text { subtropical }\end{array}$ & $\begin{array}{l}\text { Humid } \\
\text { tropical }\end{array}$ & $\begin{array}{l}\text { Humid } \\
\text { tropical }\end{array}$ & Temperate & Mediterranean \\
\hline Host species & $\begin{array}{l}P . \\
\text { dipterocarpacea, } \\
\text { Aldina sp. }\end{array}$ & $\begin{array}{l}\text { Quercus } \\
\text { crassifolia, } \\
\text { Quercus } \\
\text { laurina }\end{array}$ & $\begin{array}{l}\text { Coccoloba sp., } \\
\text { Guapira sp., } \\
\text { Neea sp. }\end{array}$ & $\begin{array}{l}\text { Dicymbe sp., } \\
\text { Aldina sp. }\end{array}$ & $\begin{array}{l}\text { Picea abies, } \\
\text { Tilia cordata, } \\
\text { Betula } \\
\text { pendula, } \\
\text { Populus } \\
\text { tremula }\end{array}$ & $\begin{array}{l}\text { Quercus } \\
\text { douglasii, } \\
\text { Quercus } \\
\text { wislizeni }\end{array}$ \\
\hline $\begin{array}{l}\text { Soil core } \\
\text { volume }\left(\mathrm{cm}^{3}\right)\end{array}$ & 125 & 942 & 2250 & 1000 & 125 & 900 \\
\hline $\begin{array}{l}\text { Number of } \\
\text { root tips } \\
\text { processed/ } \\
\text { soil core }\end{array}$ & $\begin{array}{l}\text { All root tips } \\
\text { in the core }\end{array}$ & 100 & $\begin{array}{l}\text { All root tips } \\
\text { in the core }\end{array}$ & 20 & $\begin{array}{l}\text { All root tips } \\
\text { in the core }\end{array}$ & 100 \\
\hline Replicates & 9 & 80 & 60 & 57 & 108 & 64 \\
\hline $\begin{array}{l}\text { EcM tips } \\
\text { sampling } \\
\text { strategy }\end{array}$ & Morphotyping* & $\begin{array}{l}\text { Random } \\
\text { selection** }\end{array}$ & Morphotyping & gMorphotyping & gorphotyping & $\begin{array}{l}\text { Random } \\
\text { selection }\end{array}$ \\
\hline $\begin{array}{l}\text { EcM species } \\
\text { density } \\
\text { (species in } \\
\text { soil core) }\end{array}$ & $\begin{array}{l}1-6.6 \pm 3.4 \\
(\mathrm{M} \pm \mathrm{STD})-12\end{array}$ & $6.2(\mathrm{M})$ & $1.42(\mathrm{M})$ & $9-18$ & $\begin{array}{l}3.65 \pm 1.7 \\
(\mathrm{M} \pm \mathrm{STD})\end{array}$ & $6.5(\mathrm{M})$ \\
\hline
\end{tabular}

*root sorting in morphotypes before molecular analysis.

**molecular analysis of root tips randomly selected in soil core.

$\mathrm{m}=$ mean, $\mathrm{STD}=$ standard deviation

Table 3. Comparison of observed EcM species density between Pakaraimaea dipterocarpacea forest and recent tropical and temperate surveys 
Tropical

Pakaraimaea/Aldina $400 \mathrm{~m}^{2}$

Mixed* 40

This study

Mixed Dipterocarpaceae forest in $400 \mathrm{~m}^{2}$

EcM 4-26

Peay et al. (2010)

Borneo

Temperate

Arctostaphylos/Douglas fir

$\begin{array}{lll}625 \mathrm{~m}^{2} & \text { Mixed } & >40 \\ 500 \mathrm{~m}^{2} & \text { Mixed } & 25 \\ 625 \mathrm{~m}^{2} & \text { EcM } & 20 \\ 625 \mathrm{~m}^{2} & \text { EcM } & 7\end{array}$

From Horton \&

Norway spruce

Bishop pine

$625 \mathrm{~m}^{2} \quad \mathrm{EcM} \quad 7$

Bruns (2001)

Bishop pine

*mixed fruitbody and EcM survey.

Table 4. Observed EcM fungi richness in similar sized tropical and temperate forest plots

\begin{tabular}{llll}
$\begin{array}{l}\text { Fungal species } \\
\text { (accession No) } \\
\text { (host plant) }\end{array}$ & $\begin{array}{l}\text { Best aligned sequence } \\
\text { (Accession No.) } \\
\text { (overlapping base pairs/ } \\
\text { total aligned bases) }\end{array}$ & Geographic origin & $\begin{array}{l}\text { Host family } \\
\text { (when tropical) }\end{array}$ \\
\hline
\end{tabular}

Agaricales

Amanita BM08C22 EcM 780

$\left(\mathrm{Pd}^{*}\right)$

(JN168679)

Neotropical, Guyana

Fabaceae

(627/628, 99\%)

Amanita BM07C21 Amanita vaginata

(AB458889)

$(433 / 508,85 \%)$

Amanita BM07C1 Amanita populiphila

(HQ539724)

(719/751, 96\%)

Cortinarius BM08C20Cortinarius limonius

$\left(\mathrm{Pd}^{*}, \mathrm{~A}^{\delta}\right)$

(GQ159869)

(364/412, 88\%)

Cortinarius BM07C2 Uncultured ectomycorrhizal

$\left(\mathrm{Pd}^{*}\right)$

fungus

L7789_Cort MAD02

(FR731470)

(697/736, 95\%)

Cortinarius BM08C11Uncultured ectomycorrhizal

$\left(\mathrm{Pd}^{*}\right)$

fungus

L7789_Cort MAD02

Paleotropical,

(Caesalpiniaceae,

Papilionoideae)

Thailand

Dipterocarpaceae

North temperate

North temperate

(FR731470)

$(724 / 761,95 \%)$

Cortinarius BM08C30Uncultured ectomycorrhiza cloneNorth temperate

SDL13

(FJ769528)

$(506 / 548,92 \%)$

Cortinarius 1PdM4 Uncultured ectomycorrhizal

Paleotropical,

Not given

Madagascar

Paleotropical,

Madagascar

Not given

(JQ063044)

$\left(\mathrm{Pd}^{\circ}\right)$

fungus isolate

Paleotropical ,

Fabaceae

Cameroon

(Caesalpiniaceae), L5110_Cort_Cam02†

Phyllantaceae 


\begin{tabular}{|c|c|c|c|}
\hline $\begin{array}{l}\text { Fungal species } \\
\text { (accession No) } \\
\text { (host plant)\$ }\end{array}$ & $\begin{array}{l}\text { Best aligned sequence } \\
\text { (Accession No.) } \\
\text { (overlapping base pairs/ } \\
\text { total aligned bases) }\end{array}$ & Geographic origin & $\begin{array}{l}\text { Host family } \\
\text { (when tropical) }\end{array}$ \\
\hline & $\begin{array}{l}\text { (FR731783) } \\
(590 / 623,95 \%)\end{array}$ & & \\
\hline $\begin{array}{l}\text { Inocybe BM08C27 } \\
\left(\mathrm{JQ}^{\circ} 63045\right) \\
\left(\mathrm{Pd}^{\circ}, \mathrm{A}^{\circ}\right)\end{array}$ & $\begin{array}{l}\text { Inocybe EcM } 434 \\
\text { (JN168723) } \\
(586 / 669,88 \%)\end{array}$ & Neotropical, Guyana & $\begin{array}{l}\text { Fabaceae } \\
\text { (Caesalpiniaceae, } \\
\text { Papilionoideae) }\end{array}$ \\
\hline $\begin{array}{l}\text { Inocybe BM03M2 } \\
(\mathrm{JQ063046)} \\
\left(\mathrm{Pd}^{\circ}, \mathrm{A}^{8}\right)\end{array}$ & $\begin{array}{l}\text { Inocybe EcM } 1091 \\
\text { (JN168720) } \\
(372 / 381,98 \%)\end{array}$ & Neotropical, Guyana & $\begin{array}{l}\text { Fabaceae } \\
\text { (Caesalpiniaceae, } \\
\text { Papilionoideae) }\end{array}$ \\
\hline $\begin{array}{l}\text { Tricholomataceae } \\
\text { BM08C2 }\end{array}$ & $\begin{array}{l}\text { Catathelasma ventricosum } \dagger \\
\text { (AM946418) } \\
(981 / 1088,90 \%)\end{array}$ & North temperate & \\
\hline $\begin{array}{l}\text { Boletales } \\
\text { Boletaceae } \\
\text { BM06M3 } \\
(\mathrm{JQ063047)} \\
\left(\mathrm{Pd}^{*}\right)\end{array}$ & $\begin{array}{l}\text { Boletellus ananas voucher TH6264 } \\
\text { (JN168685) } \\
(198 / 198,100 \%)\end{array}$ & Neotropical, Guyana & $\begin{array}{l}\text { Fabaceae } \\
\text { (Caesalpiniaceae, } \\
\text { Papilionoideae) }\end{array}$ \\
\hline $\begin{array}{l}\text { Boletaceae } \\
\text { BM08C29 }\end{array}$ & $\begin{array}{l}\text { Tylopilus ballouii voucher R.E. } \\
\text { Halling 8187† } \\
\text { (EU430732) } \\
(647 / 673,96 \%)\end{array}$ & $\begin{array}{l}\text { Neotropical, } \\
\text { Costa Rica }\end{array}$ & Fagaceae \\
\hline $\begin{array}{l}\text { Boletaceae } \\
\text { BM08C31 }\end{array}$ & $\begin{array}{l}\text { Austroboletus rostrupii TH8189 } \\
\text { (JN168683) } \\
(537 / 537,100 \%)\end{array}$ & Neotropical, Guyana & $\begin{array}{l}\text { Fabaceae } \\
\text { (Caesalpiniaceae, } \\
\text { Papilionoideae) }\end{array}$ \\
\hline $\begin{array}{l}\text { Boletaceae } \\
\text { BM08C8 }\end{array}$ & $\begin{array}{l}\text { Tylopilus felleus } \dagger \\
\text { (AY586723) } \\
(734 / 772,95 \%)\end{array}$ & North temperate & \\
\hline $\begin{array}{l}\text { Boletaceae } \\
7 \mathrm{MM} 7 \\
(\mathrm{JQ} 063048) \\
\left(\mathrm{Pd}^{\circ}\right)\end{array}$ & $\begin{array}{l}\text { Boletus sp. MHM075 } \\
\text { (EU569236) } \\
(252 / 280,90 \%)\end{array}$ & Neotropical, Mexico & Fagaceae \\
\hline $\begin{array}{l}\text { Scleroderma } \\
\text { BM08C9 } \\
\text { Cantharellales }\end{array}$ & Unsequenced & & \\
\hline $\begin{array}{l}\text { Cantharellus } \\
\text { BM07C6 }\end{array}$ & $\begin{array}{l}\text { Cantharellus cibarius } \\
\text { (AB453024) } \\
(236 / 251,94 \%)\end{array}$ & $\begin{array}{l}\text { Paleotropical, } \\
\text { Thailand }\end{array}$ & Dipterocarpaceae \\
\hline $\begin{array}{l}\text { Clavulina } \\
\text { BM03sp1 } \\
\left(\mathrm{JQ}^{\circ} 63049\right) \\
\left(\mathrm{Pd}^{\circ}, \mathrm{A}^{8}\right)\end{array}$ & $\begin{array}{l}\text { Clavulina voucher MCA } 4022 \\
\text { (MCA4022) } \\
(541 / 545,99 \%)\end{array}$ & Neotropical, Guyana & $\begin{array}{l}\text { Fabaceae } \\
\text { (Caesalpiniaceae) }\end{array}$ \\
\hline Clavulina BM07C10 & $\begin{array}{l}\text { Clavulina amazonensis isolate } \\
\text { TH9191 } \\
\text { (HQ680356) } \\
(596 / 650,92 \%)\end{array}$ & Neotropical, Guyana & $\begin{array}{l}\text { Fabaceae } \\
\text { (Caesalpiniaceae) }\end{array}$ \\
\hline $\begin{array}{l}\text { Clavulina BM07C9 } \\
\left(\mathrm{Pd}^{\delta}\right)\end{array}$ & $\begin{array}{l}\text { Clavulina humicola } \\
\text { (DQ056368) } \\
(555 / 590,94 \%)\end{array}$ & Neotropical, Guyana & $\begin{array}{l}\text { Fabaceae } \\
\text { (Papilionoideae) }\end{array}$ \\
\hline
\end{tabular}




\begin{tabular}{|c|c|c|c|}
\hline $\begin{array}{l}\text { Fungal species } \\
\text { (accession No) }^{\text {(host plant) }}{ }^{\S}\end{array}$ & $\begin{array}{l}\text { Best aligned sequence } \\
\text { (Accession No.) } \\
\text { (overlapping base pairs/ } \\
\text { total aligned bases) }\end{array}$ & Geographic origin & $\begin{array}{l}\text { Host family } \\
\text { (when tropical) }\end{array}$ \\
\hline$\overline{\text { Clavulina BM08C24 }}$ & $\begin{array}{l}\text { Clavulina monodiminutiva } \\
\text { (DQ056365) } \\
(570 / 643,89 \%)\end{array}$ & Neotropical, Guyana & $\begin{array}{l}\text { Fabaceae } \\
\text { (Caesalpiniaceae, } \\
\text { Papilionoideae) }\end{array}$ \\
\hline $\begin{array}{l}\text { Hydnum BM03M19 } \\
\left(\mathrm{JQ}^{2} 63050\right) \\
\left(\mathrm{Pd}^{*}\right)\end{array}$ & $\begin{array}{l}\text { Hydnum repandum } \\
\text { (AY817136) } \\
(762 / 762,100 \%)\end{array}$ & North temperate & \\
\hline \multicolumn{4}{|l|}{ Hymenochaetales } \\
\hline $\begin{array}{l}\text { Hymenochaetales } \\
\text { 2PdM2 } \\
\left(\mathrm{JQ}^{2} 063051\right) \\
\left(\mathrm{Pd}^{\delta}, \mathrm{A}^{\delta}\right)\end{array}$ & $\begin{array}{l}\text { Coltriciella dependens specimen } \\
\text { voucher TU103611 } \\
\text { (AM412254) } \\
(735 / 802,92 \%)\end{array}$ & $\begin{array}{l}\text { Paleotropical, } \\
\text { Seychelles }\end{array}$ & $\begin{array}{l}\text { Fabaceae } \\
\text { (Caesalpiniaceae), } \\
\text { Dipterocarpaceae }\end{array}$ \\
\hline $\begin{array}{l}\text { Hymenochaetales } \\
\text { 1MM1 } \\
\text { (JQ063052) }\end{array}$ & $\begin{array}{l}\text { Coltriciella dependens specimen } \\
\text { voucher TU } 103611 \\
\text { (AM412254) } \\
(715 / 799,89 \%)\end{array}$ & $\begin{array}{l}\text { Paleotropical, } \\
\text { Seychelles }\end{array}$ & Dipterocarpaceae \\
\hline $\begin{array}{l}\text { Hymenochaetales } \\
\text { PD6.3 } \\
(\text { JQ063053) } \\
\left(\mathrm{Pd}^{*}\right)\end{array}$ & $\begin{array}{l}\text { Uncultured Coltricia voucher } \\
\text { EcM } 731 \\
\text { (JN168708) } \\
(442 / 567,78 \%)\end{array}$ & Neotropical, Guyana & $\begin{array}{l}\text { Fabaceae } \\
\text { (Caesalpiniaceae) }\end{array}$ \\
\hline \multicolumn{4}{|l|}{ Russulales } \\
\hline Russula BM07C7 & $\begin{array}{l}\text { Uncultured ectomycorrhizal } \\
\text { fungus isolate L6171_Russ_Y04 } \\
\text { (FN557554) } \\
(550 / 570,96 \%)\end{array}$ & Neotropical, Ecuador & Nyctaginaceae \\
\hline $\begin{array}{l}\text { Russula BM07C18 } \\
\left(\mathrm{Pd}^{*}, \mathrm{~A}^{*}\right)\end{array}$ & $\begin{array}{l}\text { Uncultured ectomycorrhiza } \\
\text { voucher } 403 \\
(\text { AY } 667427) \\
(556 / 583,95 \%)\end{array}$ & Neotropical, Ecuador & Nyctaginaceae \\
\hline $\begin{array}{l}\text { Russulaceae 8PdM1 } \\
\text { (JQ063054) } \\
\left(\mathrm{Pd}^{\circ}, \mathrm{A}^{\circ}\right)\end{array}$ & $\begin{array}{l}\text { Uncultured ectomycorrhizal } \\
\text { fungus isolate L7612_Russ } \\
\text { MAD41 } \\
\text { (FR731334) } \\
(236 / 261,90 \%)\end{array}$ & $\begin{array}{l}\text { Paleotropical , } \\
\text { Madagascar }\end{array}$ & Not given \\
\hline $\begin{array}{l}\text { Russulaceae 1PdM3 } \\
\left(\mathrm{JQ063055)}^{\circ}\right. \\
\left(\mathrm{Pd}^{\circ}\right)\end{array}$ & $\begin{array}{l}\text { Uncultured Russula voucher } \\
\text { EcM1094 } \\
\text { (JN168741) } \\
(225 / 245,92 \%)\end{array}$ & Neotropical, Guyana & $\begin{array}{l}\text { Fabaceae } \\
\text { (Caesalpiniaceae, } \\
\text { Papilionoideae) }\end{array}$ \\
\hline $\begin{array}{l}\text { Russula "lilac" } \\
\text { BM07C20 } \\
\text { Sebacinales }\end{array}$ & Unsequenced & & \\
\hline $\begin{array}{l}\text { Sebacinales } \\
\text { BM03M3 } \\
(\mathrm{JQ} 063056) \\
\left(\mathrm{Pd}^{\circ}, \mathrm{A}^{\circ}\right)\end{array}$ & $\begin{array}{l}\text { Sebacinales EcM } 17 \\
\text { (JN168754) } \\
(532 / 549,97 \%)\end{array}$ & Neotropical, Guyana & $\begin{array}{l}\text { Fabaceae } \\
\text { (Caesalpiniaceae, } \\
\text { Papilionoideae) }\end{array}$ \\
\hline
\end{tabular}




\begin{tabular}{|c|c|c|c|}
\hline $\begin{array}{l}\text { Fungal species } \\
\text { (accession No) } \\
\text { (host plant) })^{\S}\end{array}$ & $\begin{array}{l}\text { Best aligned sequence } \\
\text { (Accession No.) } \\
\text { (overlapping base pairs/ } \\
\text { total aligned bases) } \\
\end{array}$ & Geographic origin & $\begin{array}{l}\text { Host family } \\
\text { (when tropical) }\end{array}$ \\
\hline $\begin{array}{l}\text { Sebacinales PD10 } \\
(\mathrm{JQ} 063057) \\
\left(\mathrm{Pd}^{*}, \mathrm{~A}^{\delta}\right)\end{array}$ & $\begin{array}{l}\text { Uncultured ectomycorrhizal } \\
\text { isolate L7604_Seb MAD02 } \\
\text { (FR731328) } \\
(518 / 568,91 \%)\end{array}$ & $\begin{array}{l}\text { Paleotropical, } \\
\text { Madagascar }\end{array}$ & Not given \\
\hline $\begin{array}{l}\text { Sebacinales 6MM2 } \\
\left(\mathrm{JQ}^{2} 63058\right) \\
\left(\mathrm{Pd}^{\circ}, \mathrm{A}^{\circ}\right) \\
\text { Thelephorales }\end{array}$ & $\begin{array}{l}\text { Sebacinales voucher EcM84 } \\
\text { (JN168756) } \\
(251 / 258,97 \%)\end{array}$ & Neotropical, Guyana & $\begin{array}{l}\text { Fabaceae } \\
\text { (Caesalpiniaceae) }\end{array}$ \\
\hline $\begin{array}{l}\text { Tomentella 7AM7 } \\
\text { (JQ063059) } \\
\left(\mathrm{A}^{\circ}\right)\end{array}$ & $\begin{array}{l}\text { Tomentella EcM } 963 \\
\text { (JN168770) } \\
(342 / 358,96 \%)\end{array}$ & Neotropical, Guyana & $\begin{array}{l}\text { Fabaceae } \\
\text { (Caesalpiniaceae) }\end{array}$ \\
\hline $\begin{array}{l}\text { Tomentella PD2_3 } \\
\text { JQ063060 } \\
\left(\mathrm{Pd}^{*}, \mathrm{~A}^{\circ}\right)\end{array}$ & $\begin{array}{l}\text { Tomentella EcM } 963 \\
\text { (JN168770) } \\
(593 / 602,99 \%)\end{array}$ & Neotropical, Guyana & $\begin{array}{l}\text { Fabaceae } \\
\text { (Caesalpiniaceae, } \\
\text { Papilionoideae) }\end{array}$ \\
\hline $\begin{array}{l}\text { Tomentella PD11 } \\
(\mathrm{JQ063061)} \\
\left(\mathrm{A}^{\circ}\right)\end{array}$ & $\begin{array}{l}\text { Tomentella EcM } 712 \\
\text { (JN168764) } \\
(600 / 602,99 \%)\end{array}$ & Neotropical, Guyana & $\begin{array}{l}\text { Fabaceae } \\
\text { (Caesalpiniaceae, } \\
\text { Papilionoideae) }\end{array}$ \\
\hline $\begin{array}{l}\text { Tomentella PD9_2 } \\
\text { (JQ063062 } \\
\left(\mathrm{A}^{\circ}\right)\end{array}$ & $\begin{array}{l}\text { Tomentella EcM712 } \\
\text { (JN168764) } \\
(587 / 605,97 \%)\end{array}$ & Neotropical, Guyana & $\begin{array}{l}\text { Fabaceae } \\
\text { (Caesalpiniaceae, } \\
\text { Papilionoideae) }\end{array}$ \\
\hline Tomentella BM07C39 & $\begin{array}{l}\text { 9 Tomentella MES348 } \\
\text { (JN168772) } \\
(170 / 174,98 \%)\end{array}$ & Neotropical, Guyana & $\begin{array}{l}\text { Fabaceae } \\
\text { (Caesalpiniaceae, } \\
\text { Papilionoideae) }\end{array}$ \\
\hline $\begin{array}{l}\text { Tomentella 2PdM6 } \\
\text { (JQ063063) } \\
\left(\mathrm{Pd}^{\delta}\right)\end{array}$ & $\begin{array}{l}\text { Tomentella EcM } 698 \\
\text { (JN168763) } \\
(318 / 329,97 \%)\end{array}$ & Neotropical, Guyana & $\begin{array}{l}\text { Fabaceae } \\
\text { (Caesalpiniaceae, } \\
\text { Papilionoideae) }\end{array}$ \\
\hline
\end{tabular}

†LSU sequence. Unmarked aligned sequences include ITS region.

${ }^{\S}$ Host for each fungal OTU was identified from EcM after $r b c \mathrm{~L}$ sequencing ${ }^{\circ}$, root tracing* ${ }^{*}$ or root morphology ${ }^{\delta}$. Fungal OTUs with no identified host were collected only from the fruiting bodies. $\mathrm{Pd}: P$. dipterocarpacea; A : Aldina sp. B.

Table 5. Sequence matches of ectomycorrhizas and ectomycorrhizal fruit bodies collected in Pakaraimaea dipterocarpacea forest

\subsection{Inocybe EcM frequency and occurrence on host plants in the plot}

Two Inocybe species were recovered in the plot. Inocybe EcM were characteristic and could be distinguished from other fungal genera on the basis of morphology and anatomy. They were both characterized by a smooth and whitish mantle with frequent rhizomorphs and a pinkish color at the apex (Fig. 2a). Hyphae in the rhizomorphs were uniformly shaped and agglutinated (type B in Agerer 2006). EcM habit corresponded to medium-distance smooth exploration type in Agerer's (2001) EcM mycelial system. Typical Inocybe prominent clamp connections (Fig. 2b) were observed in the rhizomorphs.

Despite the low number of Inocybe species in the total EcM fungal diversity, Inocybe EcMs were frequent and were observed in $55 \%$ of the soil cores. Two Inocybe species were 
recovered from traced roots of $P$. dipterocarpacea and the host identity was confirmed molecularly. They were also recovered from Aldina sp. roots in soil cores and Aldina rbcL could be sequenced for one Inocybe EcM species.

\subsection{Phylogeny and biogeography of Inocybe species associated with P. dipterocarpacea}

One Inocybe species from P. dipterocarpacea forest (BM03M2) matched (372/381, 98\% similarity) Inocybe EcM1091 ITS1/5.8S/ITS2 recovered from Aldina insignis and Dicymbe alstonii (Table 5). Phylogenetic analysis including LSU, together with fruit bodies' morphological comparisons, are necessary to confirm that EcM1091 and BM03M2 are conspecific. No significant match could be found for the second Inocybe species (Table 5). Best match with 25S LSU in GenBank was with I. epidendron (EU569840) for both species. Preliminary alignment of the two Inocybe species to Matheny et al. (2009) global Inocybaceae data set confirmed their position in Inocybe subgenus Inocybe (data not shown). These results were consistent with the characteristic Inocybe s. str. nodulose spores and pleurocystidia observed on Inocybe BM08C27 fruitbody species. LSU alignments with Matheny et al. (2009) global Inocybe subgenus Inocybe data set demonstrated that the two species were phylogenetically close to neotropical Inocybe species associated with Dicymbe and Aldina sp. in Guyana (Fig. 3). These species clustered within a tropical only, basal clade. A weakly supported node suggested a sister relationship with an African strain from Zambia. This clade was sister with a couple of related species from Zambia and Guyana.

\section{Discussion}

\subsection{EcM community diversity and structure in $P$. dipterocarpacea forest}

This is the first above and below ground EcM fungal diversity survey in a Neotropical forest dominated by a Dipterocarpaceae. Only few pioneering surveys have been reported on EcM fungal communities in Neotropical monospecific Dipterocarpaceae genera Pseudomonotes and Pakaraimaea (Franco-Molano et al., 2005; Moyersoen, 2006; Vasco-Palacios et al., 2005). Moyersoen (2006) study mostly focused on P. dipterocarpacea EcM status and only a small proportion of EcM community was described. The present study was necessary to get a more precise EcM diversity estimate in Pakaraimaea forest.

Despite the relatively small plot size, a great number of EcM fungal species was recorded. Total above and below ground species richness and species density in soil cores was comparable to highly diverse EcM temperate forest communities. It has been suggested that EcM diversity in tropical forests is lower than in temperate forests (Tedersoo \& Nara, 2010; Tedersoo et al., 2011). In contrast, with boreal forests, where a niche differentiation of EcM fungi is observed at the level of soil horizons (Rosling et al., 2003; Tedersoo et al., 2003), a study by Tedersoo et al. (2011) suggested that EcM communities are homogeneous across soil horizons in the African forests. Recent studies indicate that trends in EcM fungal biodiversity are not simply driven by a latitudinal gradient (Smith et al., 2011). Biodiversity depends on the forest type under study (Brearly, 2011; Smith et al., 2011) and is also influenced by host identity (Haug et al., 2005; Tedersoo et al., 2010a). Amongst the EcM surveys in Dipterocarpaceae vegetation (see Brearly, 2011 review and references therein), comparisons could be made with a SE Asian Mixed-dipterocarp forest (Peay et al., 2010), where diversity was measured in plot size identical to this study. EcM 
richness (Table 2) was at the top end of observed values (Table 4) in SE Asia and total above and below ground richness (Table 4) was within the range of estimated richness (9.3-60.4) in Mixed-dipterocarp forest. This comparison still needs to be taken with caution since root sampling strategy differed between the two studies. Contrary to the relatively low species density observed on Neotropical Pisonieae (Tedersoo et al., 2010a), density in $P$. dipterocarpacea was within the range or greater than in the temperate forests (Table 3). Both total fungal diversity and species density indicated that EcM fungi occur at a fine scale in $P$. dipterocarpacea forest such as in boreal forests. A greater EcM fungal diversity might be expected by sampling both organic and mineral soil layers in $P$. dipterocarpacea forest. This study extends the findings of a previous survey in a Neotropical Fabaceae forest where great levels of EcM fungi biodiversity were observed (Smith et al. 2011).

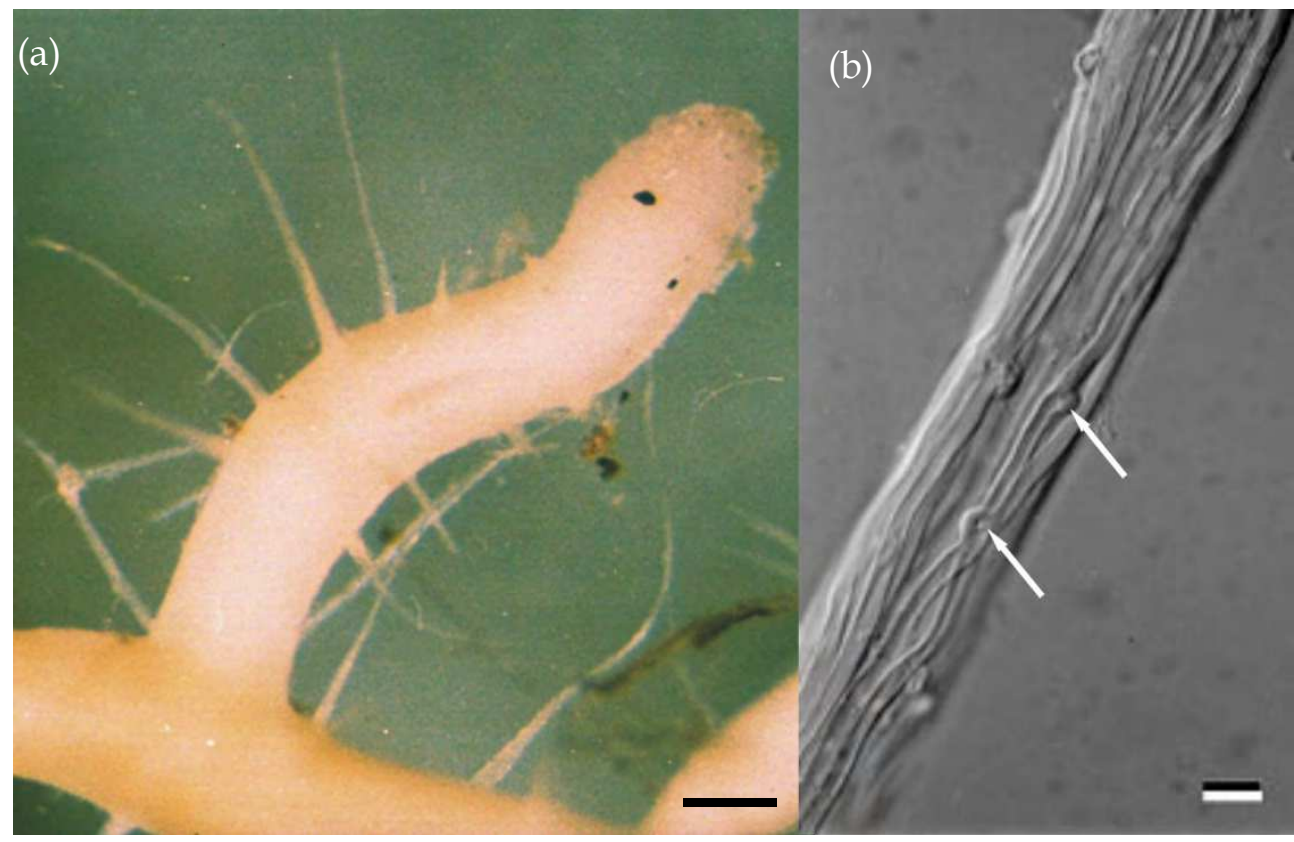

Fig. 2. (a): Habit of Inocybe sp. (BM03M2) ectomycorrhiza. Bar, 0.2 mm. (b): Detail of rhizomorph with clamp connections. Bar, $10 \mu \mathrm{m}$.

EcM fungi in the $P$. dipterocarpacea forest belong to fungal orders generally observed in the tropical forests and elsewhere (e.g. Dickie \& Moyersoen, 2008; Diédhiou et al., 2010; Lee et al., 2003; Peay et al., 2010; Riviere et al., 2007; Smith et al., 2011; Tedersoo et al., 2011). Species rich fungal orders included Agaricales, Thelephorales, Cantharellales, Boletales and Russulales. Particularly relevant was the number of Clavulina species. Many Clavulina species were also observed in Dicymbe forests and Smith et al. (2011) hypothesized that the Guayana region might be a centre of diversification for the Clavulina lineage. Cortinarius is usually considered to be rare in the tropical environments (Tedersoo et al., 2010b) and the great number of species identified in this study was striking. Interestingly, Peay et al. (2010) observed a greater number of Cortinarius in Mixed-dipterocarp forests on sandy soils. $P$. dipterocarpacea typically grows on 
white sands (Maguire \& Steyermark, 1981). Whether preference for sandy soils is an important ecological character in these tropical Cortinarius species is worth investigating. Tricholomataceae fruit body species in this study did not match any RPB2/LSU/ITS sequences either deposited in GenBank or from a recent Dicymbe forest EcM survey. This fruit body species is currently under further investigation and is potentially a new endemic species. Cortinarius, Amanita and Russula are typical "late stage" fungi characteristic of undisturbed forest stands in the temperate forests (Deacon \& Fleming, 1992; Nara et al., 2003).

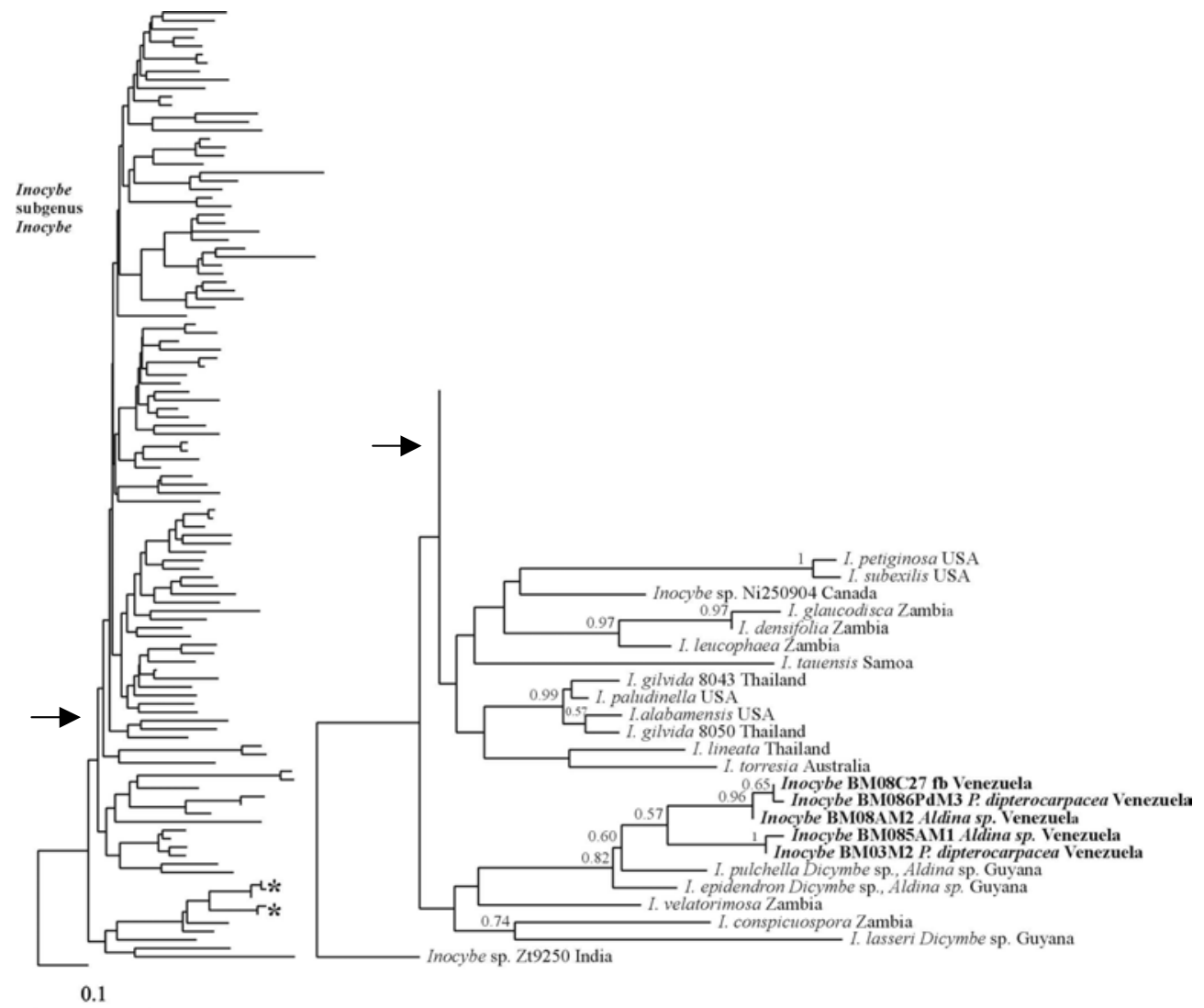

Fig. 3. Phylogenetic placement of two $P$. dipterocarpacea forest Inocybe species $\left(^{*}\right)$. Best ML tree based on combined analysis of RPB1, RPB2 and LSU sequences, resulting from 1000 replicates Rapid Bootstrapping algorithm and ML tree search in RAxML (v. 7.2.6) using GTRCAT approximation. The tree was rooted with Inocybe sp. ZT 9250. Bootstrap values < $50 \%$ are not shown.

The great total diversity, together with the abundance of these late stage fungi and the presence of a possible novel endemic species, indicated that the plot is a well preserved $P$. dipterocapacea stand despite human activities in the region. This study also indicated that Neotropical Pakaraimaea and Dicymbe forests show similarities in EcM fungal community structure. 


\subsection{EcM fungal communities comparison between $P$. dipterocarpacea and other tropical tree host species}

Until recently, biodiversity information on Neotropical lowland EcM fungi mostly relied on traditional fruit body surveys (e.g. Franco-Molano et al., 2005; Henkel et al., 2002, 2005, 2011; Matheny et al., 2003; Moyersoen, 1993; Singer et al., 1983; Vasco-Palacios et al., 2005) with inherent difficulties to confirm the host plant identity. Relatively few EcM surveys have included molecular host identifications (Haug et al., 2004; Moyersoen, 2006; Smith et al., 2011; Tedersoo et al., 2010a). This study in P. dipterocarpacea forest provides the first report of multi-host EcM fungal species associated both with a Dipterocarpaceae and EcM Fabaceae in the Neotropics.

With the exception of Pisonieae and Gnetum sp. where patterns of host specificity were reported (Bechem \& Alexander, 2011; Haug et al., 2005; Tedersoo et al., 2010a), recent tropical EcM biodiversity surveys have shown that co-occurring (sympatric) tree species including different tree families can be colonized by identical dominant EcM fungi (Diédhiou et al., 2010; Smith et al., 2011; Tedersoo et al., 2011). These sympatric tree families included the Dipterocarpaceae and Caesalpiniaceae in the Paleotropics (Tedersoo et al., 2007, 2011). In addition to host overlap in the same forest, Tedersoo et al. (2011) also observed that some EcM fungal species are broadly distributed in tropical Africa. In contrast, host identity influences EcM fungal communities in the temperate forests (Ishida et al., 2007; Tedersoo et al., 2008). This study in P. dipterocarpacea forest indicated that several fungi from different fungal groups can colonise both $P$. dipterocarpacea and a sympatric Papilionoideae. Since EcM fractional colonisation and root length were not measured, sampling design did not allow to evaluate whether or not shared fungal species were true generalists (i.e. if they equally colonized the root system of both tree hosts).

The capacity of some EcM fungi to associate with Pakaraimaea and EcM Fabaceae was further demonstrated by the comparisons between Pakaraimaea forest fungal community and Dicymbe dominated forests. Around $15 \%$ of total species in P. dipterocarpacea forest (including undescribed Amanita sp. BM08C22, Boletellus ananas, Austroboletus rostrupii, Clavulina MCA4022 and two undescribed Tomentella species) were conspecific ( $\geq 99 \%$ ITS similarity) with specimens collected in Dicymbe forest $240 \mathrm{~km}$ apart from the study site. Cantharellus guianensis might be an additional example of broadly distributed species but this hypothesis should be tested with complementary morphological and molecular study. Interestingly, C. guianensis was also described in P. tropenbosii dominated forest (FrancoMolano et al., 2005). Phylogenetics and morphological comparisons are necessary to confirm that the remaining 6 presumed shared species between $P$. dipterocarpacea and Dicymbe forests are conspecific. This study demonstrated that a proportion of EcM fungi associated with $P$. dipterocarpacea can also associate with sympatric and allopatric tree species belonging to EcM Fabaceae in the same region.

\subsection{Inocybe ecology and phylogeography}

Matheny et al. (2009) suggested that Inocybe flora in the Neotropics resulted from multiple recent immigration events in South America. The lower Inocybe diversity in the Neotropics than in the Paleotropics was attributed (apart from sampling effort) to a combination of factors including competition or extinction and paucity of EcM tree hosts. 
The low proportion of Inocybe species in P. dipterocarpacea forest EcM fungal community was consistent with the general trend in the Neotropics. At least three different Inocybe lineages were observed in Dicymbe forests (Matheny et al., 2003; Matheny et al., 2009). P. dipterocaracea survey needs to be extended to test whether this tree species also associates with several Inocybe lineages.

Despite the few species recorded, the frequent occurrence of Inocybe EcM suggested that this fungal group is successful in $P$. dipterocarpacea plot. An interesting feature of morphotypes observed on two $P$. dipterocarpacea Inocybe species was the presence of rhizomorphs with agglutinated hyphae and ramified distal end in close contact with soil particles. A small proportion of Inocybe EcM morphotypes have been formally described (Agerer, 2006) and only one of them (I. avellana in association with Shorea, Dipterocarpaceae) (Ingleby, 1999) was tropical. One of the patterns in described morphotypes was the presence of emanating hyphae only as soil exploration structures (Agerer, 2006). Ryberg et al. (2010) demonstrated that soil preference reflects the evolutionary history of Inocybaceae. Preference for poor soil prevail in some phylogenetic groups. Agerer (2001) highlighted the possible ecological importance of rhizomorphs for soil exploration and plant nutrition. Peay et al. (2011) also suggested that differences in EcM exploration type influence EcM fungal dispersion ability. Rhizomorphs might contribute to the success of Inocybe in poor sandy soils associated with $P$. dipterocarpacea forest. Whether this morphological character is a conserved morphological characteristic in Inocybe subgenus Inocybe basal clade is worth investigating.

In contrast with the hypothesis that Inocybe species associated with $P$. dipterocarpacea represent an isolated immigration event (Matheny et al., 2009), both BLASTN and phylogenetic analysis demonstrated that $P$. dipterocarpacea shared identical or phylogenetically close Inocybe species with EcM Fabaceae. The capacity of two Inocybe species to associate with both $P$. dipterocarpacea and co-occurring Aldina species $\mathrm{B}$ is consistent with the host generalist status of Inocybaceae within angiosperms in the temperate forests (Ryberg et al., 2010; Kuyper, 1986). This host sharing ability was demonstrated for several Inocybe species on Dicymbe and co-occurring Aldina in the Guayana region (Smith et al., 2011). Most interestingly, Inocybe species in P. dipterocarpacea forest were phylogenetically close to species in Dicymbe dominated forest. Singer et al. (1983) described I. amazoniensis from Campinarana vegetation near Manaus, Northern Brazil. They did not identify I. amazoniensis host plant and putative EcM hosts in Campinarana include EcM Fabacaeae (Aldina), Pisonieae and Coccolobeae. Confirmation of I. amazoniensis phylogenetic position which is morphologically close to I. epidendron (Matheny et al., 2003) and Inocybe sp. BM08C27 (unpublished data) would further support the evidence that several closely related Inocybe species associate with diverse tree hosts including Dipterocarpaceae and EcM Fabaceae across the Guayana region.

The broad distribution of phylogenetically close Inocybe species might be the result of an efficient dispersion ability, the capacity to associate with different tree hosts and a long evolutionary history in the Guayana Region. A possible explanation for the disjunct distribution of Inocybe related species in the region is that extant forests with a dominance or codominance of one or several Neotropical EcM tree families including Dipterocarpaceae (Franco-Molano et al., 2005; Moyersoen, 2006; Vasco Palacios et al., 2005) and EcM Fabaceae (Henkel et al., 2002; Singer \& Araujo, 1979, 1986) are remnants of more extensive EcM forests in the past. This hypothesis is difficult to evaluate since knowledge on Guayanan Region 
vegetation history is scanty (O Huber, pers. com). Inocybe is often mentioned in the literature as a pioneer and an effective coloniser by spore dispersal (Smith and Read, 2008). Although they are often scattered in AM dominated forests (Béreau et al., 1997; Lodge, 1987; Singer \& Araujo, 1979; St John, 1980; Tedersoo et al., 2010a), species belonging to confirmed EcM tree hosts are diverse and widely distributed in the Guayana region (Table 1). These nondominant Neotropical EcM plants might have contributed to the dispersion of EcM fungal groups such as Inocybe subgenus Inocybe by co-migration (Halling, 2001) or by providing "EcM tree islands" for fungus colonization (Peay et al., 2007). Inocybe species belonging to different clades in Inocybe subgenus Inocybe (data not shown) have been found on Guapira and Neea sp. from Ecuador, Belize and US Virgin Islands (Matheny et al., 2009; Tedersoo et al., 2010a) as well as on Coccoloba species in Ecuador (Tedersoo et al., 2010a). Fruit bodies belonging to $8 \mathrm{EcM}$ lineages including Inocybaceae were reported by Moyersoen (1993) in Bana vegetation, Southern Venezuela, where Pisonieae (Neea and Guapira) are particularly conspicuous together with A. kunhardtiana and C. excelsa. Additional surveys are necessary to test the importance of non-dominant EcM tree hosts on EcM fungi dispersion and diversity in Guayana Region. Phylogeographic analyses similar to this Inocybe study should also be undertaken on more diverse fungal lineages such as Clavulina that are associated with different confirmed tree hosts from different families across the Guayana region (Henkel et al., 2005, 2011; Smith et al. 2011).

P. dipterocarpacea Inocybe clustering within a basal clade found only in the tropics demonstrated their tropical origin. The results are consistent with Matheny et al. (2009) in the species composition of the Inocybe subgenus Inocybe basal clade (tree node 84). These basal species were not included in the phylogenetic study by Kropp and Albee-Scott (2010), who selected a subset of Neotropical and Paleotropical Inocybe strains. According to molecular clock phylogeny, the estimated age of tree node 84 was $65 \mathrm{Myr}$ (41-89 Myr, 95\% CI) (Matheny et al. 2009). Inocybe species associated with $P$. dipterocarpacea are the recorded oldest origin on a Dipterocarpaceae. The present study highlights the need to get a more exhaustive Dipterocarpaceae Inocybe ITS/LSU/RPB1/RPB2 sequence data set across the tropics. Comparisons of EcM flora between P. dipterocarpacea and P. tropenbosii as well as Dipterocarpaceae from Africa or the related EcM family Sarcolanaceae (Ducousso et al., 2004) from Madagascar would be particularly appropriate. Basal clade topology fits with a vicariance hypothesis, but Matheny et al. (2009) excluded this hypothesis on the basis of an estimated divergence date. An alternative boreotropical migration route (Pennington \& Dick, 2004) was proposed (Matheny et al., 2009) but this hypothesis is not reflected in the topology of Inocybe subgenus Inocybe phylogeny. Long distance dispersal between Africa and South America would be another alternative hypothesis. There is no clear cut biogeographic interpretation for the basal clade including Paleotropical and Neotropical Inocybe subgenus Inocybe strains. Observation of two of these basal species on $P$. dipterocarpacea, a tree host with possible Gondwanic ancestors, is intriguing.

\section{Conclusion}

This study demonstrated the importance of fungal dispersion and host sharing in understanding of the EcM community associated with $P$. dipterocarpacea. Host sharing between sympatric tree species belonging to different lineages within Fabaceae had already been described in a Dicymbe dominated forest (Smith et al. 2011). This study highlighted that 
EcM fungal species can associate with allopatric tree hosts belonging to two typical EcM tree families in the Guayana region, Dipterocarpaceae and EcM Fabaceae. There is a need to extend Inocybe phylogeographic study to other fungal groups and to include non-dominant tree species including Pisoniae and Coccolobeae in the surveys in Guayana Region. These studies are necessary to evaluate the importance of host overlap in the area and the possible importance of non-dominant host tree species in EcM fungal dispersion and diversity.

The hypothesized origin of Inocybaceae is Paleotropical (Matheny et al., 2009). The old origin of Inocybe strains associated both with P. dipterocarpacea and EcM Fabaceae and their tropical root suggest a long evolutionary history of EcM symbiosis in the Neotropics. Present knowledge of Inocybaceae molecular clock phylogeny is insufficient to say with certainty which EcM radiation scenario could explain the disjunct distribution of the basal clade of Inocybe subgenus Inocybe. A more complete ITS/LSU/RPB1/RPB2 sequence data set including Inocybe strains associated with Dipterocarpaceae across the tropics is needed to improve the understanding of Dipterocarpaceae associated Inocybe phylogeography.

This study confirmed that forests dominated by typical EcM tree families host EcM rich fungal communities in the Guayana Region. EcM fungal species density was great and further studies are needed to evaluate the effect of soil substrate on EcM fungal communities. The presence of a possible tropical endemic Tricholomataceae species in the $P$. dipterocarpaceae forest suggests that EcM flora could include old relictual species, therefore confirming the great biodiversity value of EcM flora in the Guayana Region (Smith et al., 2011).

\section{Acknowledgement}

Several research institutions (Instituto Venezolano de Investigaciones Científicas, INRA Nancy, University of Liège, University of Tübingen, University of Aberdeen) provided support including Lab facilities at different stages of this research. FNRS sponsored two field trips and expenses for molecular analysis were partially funded by University of Liège and University of Aberdeen. Thanks to G. Cuenca, J. Rosales, T. Iturriaga, V. Demoulin and IJ. Alexander for their support in my research, M. Weiss for advises in phylogenetic analysis, O. Huber and K Scott for advice and comments on the manuscript and C. Rondon and K. Licea for help in the field.

\section{References}

Agerer, R. 1991. Characterisation of ectomycorrhiza. Methods in Microbiology, 23, pp. 25-73.

Agerer, R. 2001. Exploration types of ectomycorrhizae - A proposal to classify ectomycrrrhizal mycelial system according to their pattern of differentiation and putative ecological importance. Mycorrhiza, 11, pp. 107-114.

Agerer, R. 2006. Fungal relationships and structural identity of their ectomycorrhizae. Mycological Progress, 5, pp. 67-107.

Alexander, I.J. 1989. Mycorrhizas in tropical forests, In: Mineral nutrients in tropical forest and savanna ecosystems, Proctor, J, pp. 169-188, Blackwell Scientific Publications, Oxford.

Alexander, I.J. 2006. Ectomycorrhizas - out of Africa? New Phytologist, 172, pp. 589- 591.

Altschul, SF., Thomas, LM., Alejandro, AS., Jinghui, Z., Webb, M., David, JL. 1997. Gapped BLAST and PSI-BLAST: a new generation of protein database search programs. Nucleic Acids Research, 25, pp. 3389-3402. 
Bechem EET., Alexander, IJ. 2011. Mycorrhizal status of Gnetum spp. in Cameroon: evaluating diversity with a view to ameliorating domestication effort. Mycorrhiza, doi 10.1007/s00572-011-0384-0.

Béreau, M., Gazel, M., Garbaye, J. 1997. Mycorrhizal symbiosis in trees of the tropical rainforest of French Guiana. Canadian Journal of Botany, 75, pp. 711-716.

Brearly, F. 2011. Ectomycorrhizal associations of the Dipterocarpaceae in tropical forests. Biotropica, in Press.

Deacon JW., Fleming, LV. 1992. Interactions of ectomycorrhizal fungi, In: Mycorrhizal Functioning: An Integrative Plant-Fungal Process, Allen, MF, pp. 249-300, Chapman \& Hall, New York.

Dickie, IA., Moyersoen B. 2008. Towards a global view of ectomicorrhizal ecology. New Phytologist, 180, pp. 263-265.

Diédhiou, AG., Selosse, M-A., Galiana A., Diabaté M., Dreyfus B., Bâ AM., Miana de Faria S., Béna G. 2010. Multi-host ectomycorrhizal fungi are predominant in a Guinean tropical rainforest and shared between canopy trees and seedlings. Environmental Microbiology, 12( 8), pp. 2219-2232.

Ducousso, M., Béna, G., Bourgeois, C., Buyck, B., Eyssartier, G., Vincelette, M., Rabevohitra, R., Randrihasipara, L., Dreyfus, B., Prin, Y. 2004. The last common ancestor of Sarcolanaceae and Asian dipterocarp trees was ectomycorrhizal before the IndiaMadagascar separation, about 88 million years ago. Molecular Ecology, 13, pp. 231-236.

Franco-Molano, AE., Vasco Palacios, AM., Quintero CL., Boekhout, T. 2005. Macrohongos de la región del Medio Caquetá, Colombia. Guia de Campo Colombia. Multimpresos Ltda, Colombia.

Gardes, M., Bruns, TD. 1993. ITS primers with enhanced specificity for basidiomycetes application to the identification of mycorrhizae and rusts. Molecular Ecology, 2, pp. 113-118.

Halling, RE. 2001. Ectomycorrhizae: co-evolution, significance, and biogeography. Annals of the Missouri Botanical Garden, 88, pp. 5-13.

Halling, RE., Osmundson TW., Neves M-A. 2008. Pacific boletes: Implications for biogeograpic relationships. Mycological Research, 112, pp. 437-447.

Haug, I., Weiß, M., Momeier J., Oberwinkler, F., Kottke, I. 2005. Russulaceae and Thelephoraceae form ectomycorrhizas with members of the Nyctaginaceae (Caryophyllales) in the tropical mountain rainforest of southern Ecuador. New Phytologist, 165, pp. 923-936.

Henkel, TW., Terborgh, J., Vilgalys, RJ. 2002. Ectomycorrhizal fungi and their leguminous hosts in the Pakaraima mountains of Guyana. Mycological Research, 106, 5, pp. 515-531.

Henkel TW., Meszaros R., Aime MC., Kennedy A. 2005. New Clavulina species from the Pakaraima mountains of Guyana. Mycological Progress, 4, pp. 343-350.

Henkel TW., Aime MC., Ueling JK., Smith, ME. 2011. New species and distribribution records of Clavulina (Basidiomycota) from Guiana Shield. Mycologia, 103, 4, doi: 10.3852/10-355.

Horton, TR., Bruns, TD. 2001. The molecular revolution in ectomycorrhizal ecology: peeking into the black-box. Molecular Ecology, 10, pp. 1855-1871.

Ishida TA., Nara K., Hogetsu T. 2007. Host effects on ectomycorrhizal fungal communities: insight from eight host species in mixed conifer-broadleaf forests. New Phytologist, 174, pp. $430-440$. 
Ingleby K.1999. Inocybe avellana Horak + Shorea leprosila Miq. In: Description of Ectomycorrhizas 4 (Agerer R, Danielson RM, Egli S, Ingleby K, Luoma D, Treu R, eds.), pp 55-60, Einhorn-Verlag, Germany.

Käss, E., Wink, M. 1997. Phylogenetic relationships in the Papilionoideae (family Leguminosae) based on nucleotide sequences of cpDNA $(r b c \mathrm{~L})$ and ncDNA (ITS 1 and 2). Molecular Phylogenetics and Evolution, 8, 1, pp. 65-88.

Kropp, BR., Albee-Scott S. 2010, Inocybe tauensis, a new species from the Samoan Archipelago with biogeographic evidence for a paleotropical origin. Fungal Biology, 114, 9, pp. 790-796.

Kuyper, T. 1986. A revision of the genus Inocybe in Europe. I. Subgenus Inosperma and the smooth-spored species of subgenus Inocybe. Persoonia, supplement volume 3, pp. 1-247.

Lee, SS., Watling, R., Turnbull, E. 2003. Diversity of putative ectomycorrhizal fungi in Pasoh Forest Reserve, In: Pasoh: Ecology of a lowland rainforest in Southeast Asia, Okuda, T., Manokaran, N., Matsumoto, Y., Niiyama, K., Thomas, S.C., Ashton, P.S., pp. 149159, Springer, Tokyo.

Lodge, DJ. 1987. Resurvey of mycorrhizal associations in the El Verde rainforest in Puerto Rico, In: Proceedings of the $7^{\text {th }}$ North American Conference on Mycorrhizae, Sylvia, DM., Hung, LL., Graham, JH. pp. 127. Institute of Food and Agriculture Science, University of Florida, Gainsville.

Maguire, B., Ashton, PS., de Zeeuw, C., Giannasi, DE., Niklas, KJ. 1977. Pakaraimoideae, Dipterocarpaceae of the Western hemisphere. Taxon, 26, 4, pp. 341-385.

Maguire, B., Ashton, PS. 1980. Pakaraimaea dipterocarpacaea II. Taxon, 29(2/3), pp. 225-231.

Maguire, B., Steyermark, JA. 1981. Pakaraimaea dipterocarpacea. III. Memoires of the New York Botanical Garden, 32, pp. 306-309.

Matheny, PB., Aime MC., Bougher NL., Buyck B., Desjardin DE., Horak, E., Kropp BR., Lodge DJ., Soytong K., Trappe JM., Hibbett DS. 2009. Out of the Paleotropics? Historical biogeography and diversification of the cosmopolitan ectomycorrhizal mushroom family Inocybaceae. Journal of Biogeography, 36, 4, pp. 577-592.

Matheny, PB. 2005. Improving phylogenetic inference of mushrooms with RPB1 and RPB2 nucleotide sequences (Inocybe; Agaricales). Molecular Phylogenetics and Evolution, 35, pp. 1-20.

Matheny, PB., Aime, MC., Henkel, TW. 2003. New species of Inocybe from Dicymbe forests of Guyana. Mycological Research, 107(4), pp. 495-505.

Morris, MH., Pérez-Pérez, MA., Smith, ME., Bledsoe, CS. 2008. Multiple species of ectomycorrhizal fungi are frequently detected on individual oak root tips in a tropical cloud forest. Mycorrhiza, 18, pp. 375-383.

Morris, MH., Pérez-Pérez, MA., Smith, ME., Bledsoe, CS. 2009. Influence of host species on ectomycorrhizal communities associated with two co-occurring oaks (Quercus spp.) in a tropical cloud forest. FEMS Microbiology Ecology, 69, pp. 274-287.

Moyersoen, B. 1993. Ectomicorrizas y micorrizas vesículo-arbusculares en Caatinga Amazónica del Sur de Venezuela. Scientia Guaianae, 82 pp.

Moyersoen, B., Beever RE., Martin F. 2003. Genetic diversity of Pisolithus in New Zealand indicates multiple long-distance dispersal from Australia. New Phytologist, 160, pp. 569-579. 
Moyersoen, B. 2006. Pakaraimea dipterocarpacea is ectomycorrhizal, indicating an ancient Gondwanaland origin for the ectomycorrhizal habit in Dipterocarpaceae. New Phytologist, 172, pp. 753-762.

Nara K., Nakaya H., Wu, B., Zhou Z., Hogetsu, T. 2003. Underground primary succession of ectomycorrhizal fungi in a volcanic desert on Mout Fuji. New Phytologist, 159, pp. 743-756.

Nilsson RH., Kristiansson E., Ryberg M., Hallenberg N., Larsson K-H. 2008. Intraspecific ITS variability in the kingdom fungi as expressed in the international sequence databases and its implications for the molecular species identification. Evolutionary Bioinformatics, 4, pp. 193-201.

Peay KG., Bruns TD., Kennedy PG., Bergemann SE., Garbelotto, M. 2007. A strong speciesarea relationship for the eukaryotic soil microbes: island size matters for ectomycorrhizal fungi. Ecology Letters, 10, pp. 470-480.

Peay, KG., Kennedy PG., Davies SJ., Tan, S., Bruns, T. 2010. Potential link between plant and fungal distributions in a dipterocarp rainforest: community and phylogenetic structure of tropical ectomycorrhizal fungi across a plant and soil ecotone. New Phytologist, 185, pp. 529-542.

Peay, KG., Kennedy PG., Bruns T. 2011. Rethinking ectomycorrhizal succession: are root density and hyphal exploration types drivers of spatial and temporal zonation? Fungal Ecology, 4, pp. 233-240.

Pennington, RT., Dick, CW. 2004. The role of immigrants in the assembly of the South American rainforest tree flora. Philosophical Transactions Royal Society London B, 359, pp. 1611-1622.

Pirozynski KA. 1983. Pacific mycogeography: an appraisal. Australian Journal of Botany, Suppl. Ser. 10, pp. 137-159.

Rinaldi AC., Comandini O., Kuyper TW. 2008. Ectomycorrhizal fungal diversity: separating the wheat from the chaff. Fungal Diversity, 33, pp. 1-45.

Riviere T., Diedhiou AG., Diabate M., Senthilarasu G., Natarajan K., Verbeken A., Buyck B., Dreyfus B., Bena, G., Ba AM. 2007. Genetic diversity of ectomycorrhizal basidiomycetes from African and Indian tropical rainforests. Mycorrhiza, 17, pp. 415-428.

Rosling A., Landeweert R., Lindahl BD., Larsson K-H., Kuyper TW., Taylor AFS., Finlay RD. 2003. Vertical distribution of ectomycorrhizal fungal taxa in a podzol soil profile. New Phytologist, 159, pp. 775-783.

Ryberg, M., 2009. An evolutionary view of the taxonomy and ecology of Inocybe (Agaricales) with new perspective gleaned fromGenBank metadata, Ph.D. thesis, Department of Plant and Environment Sciences, University of Gothenburg.

Ryberg, M., Larsson, E, Jacobsson, S. 2010. An evolutionary perspective on morphological and ecological characters in the mushroom forming family Inocybaceae (Agaricomycotina, Fungi). Molecular Phylogenetics and Evolution, 55(2), pp. 431-442.

Singer, R. 1986. The Agaricals in Modern Taxonomy. Koeltz Scientific Books.

Singer, R., Araujo, I. 1979. Litter decomposition and ectomycorrhiza in Amazonian forests. 1. A comparison of litter decomposing and ectomycorrhizal basidiomycetes in latosol terrafirme rainforest and white sand podzol Campinarana. Acta Amazonica, 9, pp. 25-42.

Singer R, Araujo, I. 1986. Litter decomposing and ectomycorrhizal Basidiomycetes in an Igapó forest. Plant Systematics and Evolution, 153, pp. 107-117. 
Singer, R., Araujo, I., Ivory, MH. 1983. The ectotrophically mycorrhizal fungi of the neotropical lowlands, especially Central Amazonia. Beihefte zur Nova Hedwigia, 77, pp. 1-352.

Smith SE., Read, DJ. 2008. Mycorrhizal symbiosis, Academic Press, 787 pp.

Smith ME, Henkel TW, Aime MC, Fremier AK, Vilgalys R. 2011. Ectomycorrhizal fungal diversity and community structure on three co-occurring leguminous canopy tree species in a Neotropical rainforest. New Phytologist doi: 10.1111/j.14698137.2011.03844.x.

Steyermark JA, Berry P.E., Holst BK. 1995. Flora of the Venezuelan Guayana. Volume 1. Missouri Botanical Garden. St. Louis. 320 pp.

St John, TV. 1980. A survey of mycorrhizal infection in an Amazonian rainforest. Acta Amazonica, 10(3), pp. 527-533.

Tedersoo, L., Köljalg, U., Hallenberg, N., Larsson, K-H. 2003. Fine scale distribution of ectomycorrhizal fungi and roots across substrate layers including coarse woody debris in a mixed forest. New Phytologist, 159, pp. 153-165.

Tedersoo, L., Suvi T., Beaver K., Köljalg, U. 2007. Ectomycorrhizal fungi of the Seychelles: diversity patterns and host shifts from the native Vateriopsis seychellarum ( Dipterocarpaceae ) and Intsia bijuga (Caesalpiniaceae) to the introduced Eucalyptus robusta (Myrtaceae), but not Pinus caribea ( Pinaceae ). New phytologist, 175, pp. 321-333.

Tedersoo, L., Nara K. 2010. General latitudinal gradient of biodiversity is reversed in ectomycorrhizal fungi. New Phytologist, 185, pp. 351-354.

Tedersoo, L., Jairus T., Horton BM., Abarenkov K., Suvi T., Saar I., Köljalg, U. 2008. Strong host preference of ectomycorrhizal fungi in a Tasmanian wet sclerophyll forest as revealed by DNA barcoding and taxon-specific primers. New Phytologist, 180, pp. 479-490.

Tedersoo, L., Sadam A., Zambrano M., Valencia R., Bahram M. 2010a. Low diversity and high host preference of ectomycorrhizal fungi in Western Amazonia, a neotropical biodiversity hotspot. The ISME journal, 4, pp. 465-471.

Tedersoo, L., May TW., Smith ME. 2010b. Ectomycorrhizal lifestyle in fungi: global diversity, distribution, and evolution of phylogenetic lineages. Mycorrhiza, 20, pp. 217-263.

Tedersoo, L., Bahram M., Jairus T., Bechem E., Chinoya S., Mpumba R., Leal M., Randrianjohany E., Razafimandimbison S., Sadam A., Naaldel T., Köljalg, U. 2011. Spatial structure and the effects of host and soil environments on communities of ectomycorrhizal fungi in wooded savannas and rainforest of Continental Africa and Madagascar. Molecular Ecology, 20, pp. 3071-3080.

Vasco Palacios, AM., Franco-Molano AE., López-Quintero CA., Boekhout T. 2005. Macromicetes (Ascomycota, Basidiomicota) de la Region del Medio Caquetá y Amazonas. Biota Colombiana, 6(1), pp. 127-140.

Vilgalys R., Hester, M. 1990. Rapid genetic identification and mapping of enzymatically amplified ribosomal DNA from several Cryptococcus species. Journal of Bacteriology, pp. $4238-4246$.

White, TJ., Bruns, T., Lee, S., Taylor, J. 1990. Amplification and direct sequencing of fungal ribosomal RNA genes for phylogenetics, In: PCR Protocols: a Guide to Methods and Applications, Innis, MA., Gelfand, DH., Sninsky, JJ., White, TJ., pp. 315-322, Academic Press, New York. 


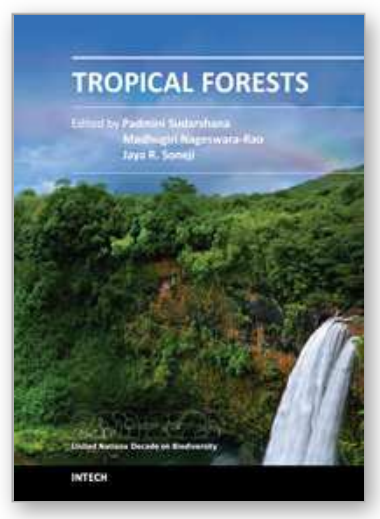

\author{
Tropical Forests \\ Edited by Dr. Padmini Sudarshana
}

ISBN 978-953-51-0255-7

Hard cover, 388 pages

Publisher InTech

Published online 14, March, 2012

Published in print edition March, 2012

The astounding richness and biodiversity of tropical forests is rapidly dwindling. This has severely altered the vital biogeochemical cycles of carbon, phosphorus, nitrogen etc. and has led to the change in global climate and pristine natural ecosystems. In this elegant book, we have defined "Tropical Forests" broadly, into five different themes: (1) tropical forest structure, synergy, synthesis, (2) tropical forest fragmentation, (3) impact of anthropogenic pressure, (4) Geographic Information System and remote sensing, and (5) tropical forest protection and process. The cutting-edge synthesis, detailed current reviews, several original data-rich case studies, recent experiments/experiences from leading scientists across the world are presented as unique chapters. Though, the chapters differ noticeably in the geographic focus, diverse ecosystems, time and approach, they share these five important themes and help in understanding, educating, and creating awareness on the role of "Tropical Forests" for the very survival of mankind, climate change, and the diversity of biota across the globe. This book will be of great use to the students, scientists, ecologists, population and conservation biologists, and forest managers across the globe.

\title{
How to reference
}

In order to correctly reference this scholarly work, feel free to copy and paste the following:

Bernard Moyersoen (2012). Dispersion, an Important Radiation Mechanism for Ectomycorrhizal Fungi in Neotropical Lowland Forests?, Tropical Forests, Dr. Padmini Sudarshana (Ed.), ISBN: 978-953-51-0255-7, InTech, Available from: http://www.intechopen.com/books/tropical-forests/dispersion-an-important-radiationmechanism-for-ectomycorrhizal-fungi-in-neotropical-lowland-forests

\section{INTECH}

open science | open minds

\section{InTech Europe}

University Campus STeP Ri

Slavka Krautzeka 83/A

51000 Rijeka, Croatia

Phone: +385 (51) 770447

Fax: +385 (51) 686166

www.intechopen.com

\section{InTech China}

Unit 405, Office Block, Hotel Equatorial Shanghai

No.65, Yan An Road (West), Shanghai, 200040, China 中国上海市延安西路65号上海国际贵都大饭店办公楼 405 单元

Phone: $+86-21-62489820$

Fax: +86-21-62489821 
(C) 2012 The Author(s). Licensee IntechOpen. This is an open access article distributed under the terms of the Creative Commons Attribution 3.0 License, which permits unrestricted use, distribution, and reproduction in any medium, provided the original work is properly cited. 